\title{
Relactação como possibilidade terapêutica na atenção a lactentes com necessidades alimentares especiais
}

\section{Relactation as therapeutical possibility in the care for infants with special food needs}

\author{
Tátila Lima de Oliveira' \\ Bibiana Arantes Moraes² \\ Lívia Lislie Ferreira Salgado ${ }^{3}$ \\ 1 Programa de Pós-graduação em Saúde da \\ Criança e do Adolescente, Faculdade de Ciências \\ Médicas. Universidade Estadual de Campinas. \\ Campinas, SP, Brasil. \\ 2 Programa de Pós-graduação em Nutrição e \\ Saúde, Faculdade de Nutrição. Universidade \\ Federal de Goiás. Goiânia, G0, Brasil. \\ ${ }^{3}$ Coordenação da Área Técnica de Nutrição. \\ Secretaria Municipal de Saúde de Rio Verde. Rio \\ Verde, GO, Brasil. \\ Correspondência / Correspondence \\ Tátila Lima de Oliveira \\ E-mail: tatila.lima@yahoo.com.br
}

\section{Resumo}

O Programa de Atenção Nutricional (PAN) de Rio Verde-GO iniciou suas atividades em 2009, financiado pelo Fundo Municipal de Saúde, visando ao atendimento de crianças diagnosticadas com intolerância à lactose, alergia à proteína do leite de vaca, soja ou múltiplas proteínas, além de erros inatos do metabolismo e desnutrição. O presente relato tem como objetivo apresentar a experiência desse programa no incentivo à amamentação por meio de acolhimento qualificado e orientações quanto à técnica de relactação. Em 2012, foram entrevistadas via telefônica 28 mães que receberam orientações para retorno da amamentação. Dentre as entrevistadas, $78,6 \%(n=22)$ fizeram a técnica e $21,4 \%$ $(\mathrm{n}=6)$ não tentaram. Entre as mães que usaram a técnica, $68,2 \%$ $(n=15)$ não retomaram a amamentação. Destas, 86,7\% $(n=13)$ alegaram que o bebê rejeitou o peito e $13,3 \%(n=2)$, que não produziram leite. Quanto ao retorno da lactação, 31,8\% (n=7) das mães que utilizaram a técnica obtiveram sucesso. O sucesso da técnica talvez possa ser ampliado com uma rede de apoio e incentivo à amamentação no município, que irá possibilitar o aumento de práticas bem-sucedidas e de baixo custo para a saúde pública, que precisam ser mais bem divulgadas à população e aos profissionais de saúde.

Palavras-chave: Lactação. Aleitamento Materno. Hipersensibilidade Alimentar. Intolerância à Lactose, Nutrição do Lactente. Sistema Único de Saúde. 


\section{Abstract}

The Nutritional Care Program (NAP) of Rio Verde-GO started its activities in 2009, supported by the Municipal Health Fund, created to attend children diagnosed with lactose intolerance, allergy to cow milk protein, soy or multiple proteins, and inborn metabolism errors and malnutrition as well. In this context, this report aims to present the experience of this program to stimulate breastfeeding through the qualified host and guidance on the technique of relactation. Twenty eight mothers who received guidelines for return of breastfeeding through the NAP were interviewed by telephone. Among the interviewed, $78.6 \%(\mathrm{n}=22)$ did the technique, $21.4 \%$ $(n=6)$ did not try. Among mothers who used the technique, $68.2 \%$ $(n=15)$ did not resume breastfeeding. Of these, $86.7 \%(n=13)$ argued that the baby rejected the breast and $13.3 \%(\mathrm{n}=2)$ produced no breastmilk. Concerning the return of lactation, $31.8 \%(n=7)$ of the mothers who used the technique succeeded. The success of the technique might be extended with a support network and encouragement for breastfeeding in town, which will increase the use of successful and low costs practices, which needs to be better disseminated to the public and healthcare professionals.

Key words: Lactation. Breastfeeding. Food Hypersensitivity. Lactose Intolerance. Infant Nutrition.

\section{Introdução}

O Programa de Atenção Nutricional (PAN) está localizado no prédio administrativo da Secretaria Municipal de Saúde de Rio Verde, um município de médio porte com cerca de 180 mil habitantes, na região sudoeste do estado de Goiás, Região CentroOeste do Brasil. ${ }^{1}$

Este programa foi criado em 2009 visando ao atendimento e fornecimento de fórmulas especiais para lactentes e crianças de primeira infância com intolerância à lactose, alergia à proteína do leite de vaca, soja ou múltiplas proteínas, além de erros inatos do metabolismo, desnutrição, doenças que comprometam o funcionamento do aparelho gastrointestinal e crianças que estejam em terapia nutricional enteral. Também recebe uma demanda de pedidos de fórmulas de partida e seguimento encaminhadas à Secretaria de Assistência Social. 
Devido à crescente demanda para o auxílio de pacientes que necessitam de continuidade na terapia nutricional após a alta hospitalar, o PAN estendeu atendimento também aos adultos com sequelas neurológicas, em tratamento oncológico, desnutridos, portadores de doenças que comprometam o funcionamento do aparelho gastrointestinal ou que estejam em terapia nutricional enteral.

Atualmente, o gasto médio anual do programa é de cerca de $\mathrm{R} \$ 1.000 .000,00 \mathrm{e}$ $65 \%(\mathrm{n}=144)$ dos usuários cadastros são crianças e $35 \%(\mathrm{n}=78)$ adultos. Das crianças cadastradas, $4 \%(n=6)$ foram atendidas por meio de mandado judicial.

No atendimento à criança, além da dispensação de fórmulas nutricionais, o programa tem o diferencial de incentivar a amamentação e promover a relactação como uma alternativa terapêutica. O inicio de tal prática ocorreu após a realização dos cursos de capacitação promovidos pelo estado em agosto de 2010. Contudo, o primeiro registro das atividades de relactação consta em agosto de 2011. Nesse período sem registro, foram realizadas orientações esporádicas e não estruturadas.

Desde 2011, o programa fornece orientação, apoio e materiais necessários para realização da técnica relactação às mães, pais ou familiares geralmente de crianças menores de seis meses que procuram o recebimento de fórmulas infantis no programa.

Pode parecer contraditório o incentivo à amamentação em uma unidade administrativa de dispensação de fórmulas infantis. Entretanto, essa prática é uma ação-chave numa cidade promotora da amamentação, pois neste serviço se concentram os casos de dificuldades de amamentação que muitas vezes poderiam ter sido evitados no pré-natal qualificado, na maternidade amiga da criança, no acompanhamento domiciliar e tão próximo da equipe de Saúde da Família, no cuidado ao puerpério realizado na atenção básica ou mesmo na rede privada ou de saúde suplementar, nas creches amigas da amamentação, entre outros pontos de atenção. ${ }^{2,3}$

Independentemente do ponto de atenção anterior, é possível que os problemas que comprometem a amamentação recairão nos pontos de dispensação de fórmulas das prefeituras, principalmente quando se diagnostica alguma necessidade de uso de fórmula especial e de alto custo. Além disso, em um programa de dispensação de fórmulas, em que o usuário apresenta prescrição médica ou de nutricionista, a ação de incentivo à amamentação pode ser interpretada por ele como resistência do programa ao fornecimento das fórmulas infantis. Desta forma, o incentivo a amamentação neste tipo de ponto de atenção, e provavelmente em outros pontos, não é possível sem que haja treinamento especifico da equipe em habilidades de comunicação. 
Para o manejo adequado da amamentação, fazem-se necessários o conhecimento técnico e o desenvolvimento de habilidades de comunicação para empoderamento da mãe. ${ }^{4-7}$ Os cursos de formação promovidos pela Secretaria de Estado da Saúde, especificamente pela Coordenação de Saúde da Criança e Coordenação de Vigilância Nutricional do Estado de Goiás - curso para Multiplicadores da Iniciativa Hospital Amigo da Criança para equipes de maternidades; curso sobre a Norma Brasileira de Comercialização de Alimentos para Lactentes e crianças de primeira infância, bicos, chupetas e mamadeiras (NBCAL); oficina para formação de tutores da Estratégia Nacional para a Alimentação Complementar Saudável (ENPACS) ${ }^{6}$ - foram primordiais para prover este conhecimento aos servidores do PAN.

A relactação, enquanto estratégia terapêutica para usuários deste e de outros programas, é uma técnica eficiente para incentivo à amamentação. Existem situações relacionadas à mãe ou ao recém-nascido em que a relactação é indicada. ${ }^{8-11}$ Para o recém-nascido, a relactação é indicada quando: está na maternidade e tem prescrição de algum líquido ou leite; deixou o peito para usar a mamadeira e sua mãe quer voltar a amamentá-lo; está com sucção pouco eficiente; com baixo ganho de peso; rejeitou uma ou duas mamas; é prematuro, está aprendendo a mamar e não consegue sugar todo o leite necessário; apresenta alguma enfermidade ou condição que o impede de fazer muito esforço (determinadas doenças neurológicas ou cardíacas) ou que acarreta hipotonia muscular (ex: síndrome de Down). ${ }^{9}$ Para as mães, essa indicação ocorre nas seguintes situações: o leite não desceu, ou ela está em pós-parto imediato; tomou medicamento para secar o leite e quer retomar a amamentação; apresenta hipogalactia (pouca produção de leite); é adotiva e deseja amamentar; ou está com a mama menor que a outra. ${ }^{9}$

No PAN, a relactação é incentivada quando ocorre alguma destas situações. Diante da observação que parte dos pais incentivados à amamentação não retornavam ao programa para busca das fórmulas infantis, resolveu-se investigar sobre o sucesso da relactação. Assim, este relato tem como objetivo apresentar a experiência do PAN no incentivo à amamentação e avaliar o sucesso da relactação em mulheres assistidas pelo Programa de Atenção Nutricional do município de Rio Verde-GO em seus primeiros oito meses de execução.

\section{Metodologia}

O presente estudo é do tipo descritivo, realizado no período de agosto de 2011 a abril de 2012, com base em 30 cadastros levantados no âmbito do PAN. 
A população-alvo eram crianças e suas respectivas mães, pais e/ou familiares que buscaram recebimento de fórmulas infantis no PAN, e foram incentivadas a realizar a técnica de relactação. Foram abordadas quanto à relactação as mães que não estavam amamentando ou estavam em aleitamento materno parcial complementado com fórmula, geralmente quando os filhos tinham idade inferior a seis meses ou quando o período de cessação da produção de leite materno era inferior a seis meses. Essa abordagem não foi realizada com mães soropositivas para HIV ou com outras doenças que impedissem a lactação e com mães que não se mostravam receptivas à retomada da lactação.

As informações sobre a técnica de relactação eram fornecidas por nutricionistas ou estagiários com treinamento em habilidades de comunicação, manejo da relactação e amamentação (pega e posição corretas, técnica do copinho, ordenha, armazenamento de leite etc.). Para isso, dispunham de material instrutivo e sondas nasogástricas $n^{\circ} 4$ para fornecimento às mães, pais ou familiares.

O material instrutivo utilizado apresentava informações sobre: as vantagens do aleitamento materno para o bebê, para a mãe, pai e família; o porquê de não serem usadas mamadeiras, chupetas ou chucas; como amamentar/técnicas (pontos chave do posicionamento e da pega adequada); como colocar o bebê no peito; quando oferecer o peito; como terminar a mamada; quando tirar o leite das mamas; como armazenar o leite materno; e relactação. O conteúdo estava distribuído em três páginas tipo folheto, com linguagem resumida e apoio de figuras.

Informações como nome dos pais e da criança, data de nascimento da criança, data da orientação, telefone dos pais e assinatura no recebimento dos materiais eram coletadas em formulário específico.

A técnica de relactação também pode ser denominada de "lactação adotiva", "indução da lactação", ou, ainda, "translactação", quando há transição da alimentação via sonda para amamentação ao seio materno. ${ }^{8-11}$ A técnica utilizada é a mesma e consiste na utilização de um dispositivo afixado no seio que está ligado e/ou mergulhado num recipiente com leite materno ou outro leite. À medida que o bebê suga, o líquido é liberado ao mesmo tempo em que a sucção estimula a hipófise na liberação de hormônios como a prolactina e ocitocina.

Esses mediadores hormonais são dependentes de estimulação mamária, por isso o bebê deve mamar sob livre demanda, inclusive durante a noite. O período para obtenção de resultados é variado; no entanto, a OMS estima que, no período de uma a seis semanas, a maioria das mulheres passa a produzir leite suficiente para alimentar o bebê sem a necessidade de complemento até os seis meses. ${ }^{7-13}$ 
A técnica, ilustrada na figura 1, consiste em: se a sonda tiver mais de um orifício, cortála, verificar se a aréola está flexível, colocar o bebê no peito, observar o posicionamento da mãe e do bebê e se a pega do bebê está correta, mergulhar a extremidade mais larga da sonda no leite. A sonda deve permanecer por cima ou na lateral do mamilo. Introduzir de 2 a $3 \mathrm{~cm}$ a ponta do cateter lentamente entre o lábio superior e aréola, em cada sugada, aproveitando o relaxamento deste lábio, cuidando para que a ponta do cateter não ultrapasse a ponta do mamilo, nem fique localizada antes. Pode-se fixar o cateter no peito da mãe com fita crepe ou esparadrapo hipoalergênico. O recipiente com leite deve ficar abaixo do nível da boca do bebê, para que o leite suba com a sucção. Se o recipiente ficar acima, o leite desce por gravidade, mesmo sem sucção. Se o bebê estiver ordenhando o leite do recipiente muito rápido, o calibre da sonda pode ser diminuído apertando-o com o dedo. ${ }^{9}$

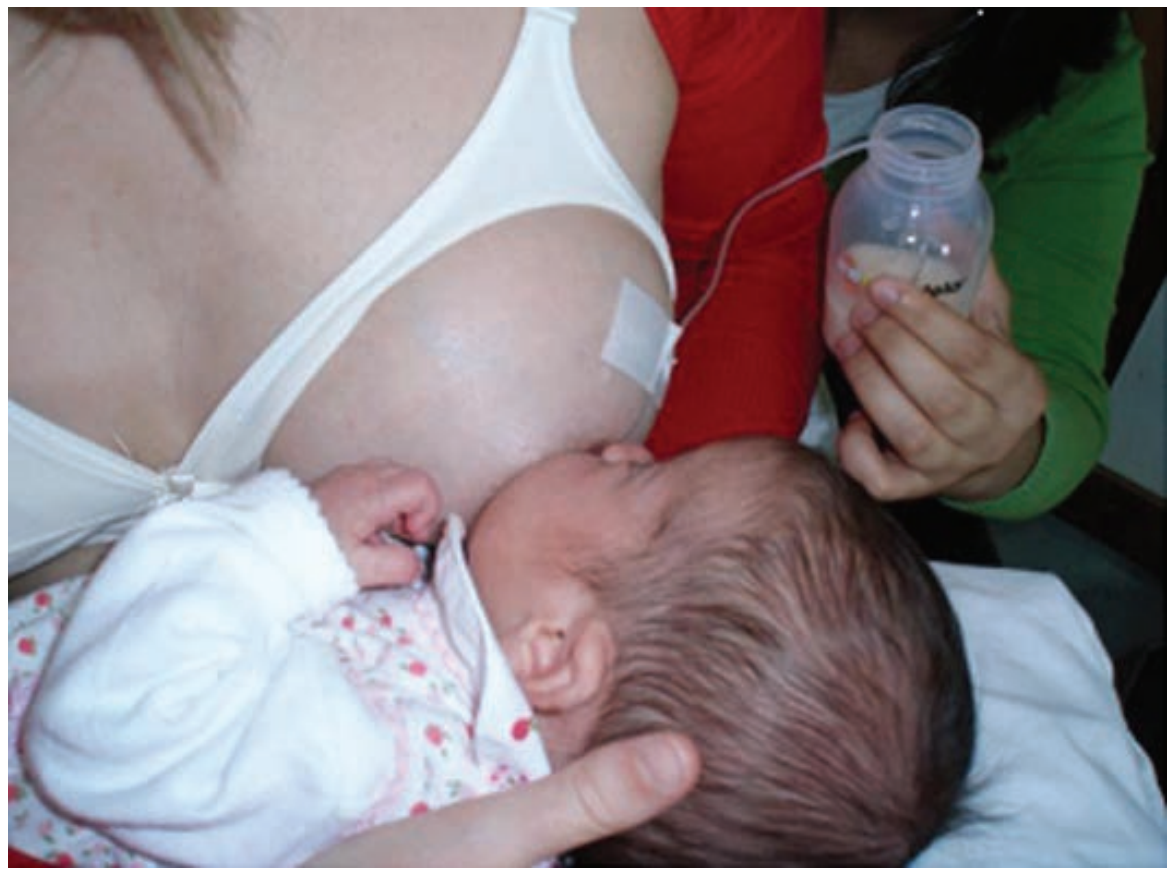

Fonte: Bordalo $(2008)^{13}$

Figura 1. Técnica de relactação com uso de sonda naso n4 e recipiente com leite. 
As entrevistas para avaliação de seguimento e realização da técnica foram realizadas no mês de abril de 2012 por meio de ligações telefônicas, quando as mães eram convidadas a responder algumas perguntas sobre as informações recebidas em relação à técnica de relactação, caso concordassem, eram-lhes feitas as seguintes perguntas: 1) Realizou a técnica?; 2) Se sim por quanto tempo, se não por que não realizou; 3) Se sim, houve retorno da produção de leite?.

A presente publicação foi submetida à aprovação como relato de experiência do serviço ao Comitê de Ética em Pesquisa com Seres Humanos da Universidade Estadual de Campinas (FCM/Unicamp) e foi aprovada no parecer CEP/ARTIGO no 008/2014 sem restrições para publicação por estar plenamente justificada, com dispensa da aplicação do Termo de Consentimento Livre e Esclarecido (TCLE).

\section{Resultados}

No período anterior à entrevista, 30 mães haviam recebido orientações sobre a técnica de relactação; destas, 93,3\% $(n=28)$ foram entrevistadas e 6,7\% $(n=2)$ não foram localizadas.

O tempo médio decorrido entre o recebimento da orientação pelas mães e familiares e a realização da entrevista foi de 3,3 meses ( \pm 2,3 desvio-padrão).

Dentre as entrevistadas, 78,6\% ( $\mathrm{n}=22)$ fizeram a técnica da relactação e 21,4\% (n=6) não tentaram. Entre as mães que usaram a técnica, $68,2 \%(n=15)$ não retomaram a produção de leite materno. Destas, $86,7 \%(\mathrm{n}=13)$ alegaram que a criança rejeitou o peito.

Das 22 mães que usaram a técnica, $31,8 \%(n=7)$ obtiveram sucesso no retorno ou aumento da lactação. No momento da entrevista, duas delas estavam em aleitamento materno exclusivo.

Ressalta-se que o sucesso da relactação neste estudo foi de 25,0\% $(n=7)$ do total de mães entrevistadas. Ou seja, uma em cada quatro mães tiveram sucesso, e cerca de uma em cada três mães que tentaram a técnica $(n=22)$ foi bem-sucedida. A figura 2 apresenta tais resultados em números absolutos. 


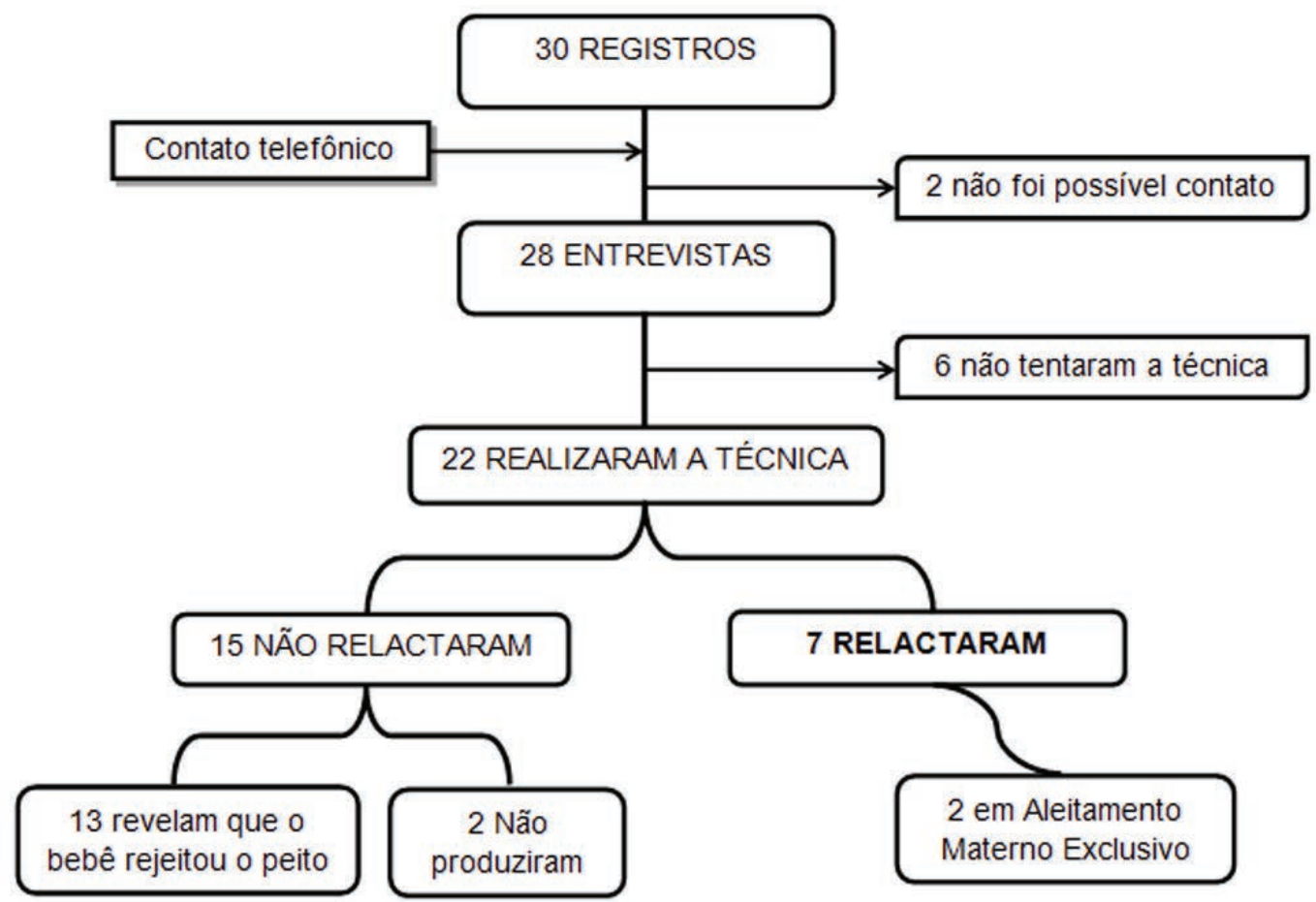

Figura 2. Fluxograma do contato com as mães que receberam informação sobre a técnica de relactação e distribuição dos desfechos observados entre aquelas que realizaram a técnica. Programa de Atenção Nutricional de Rio Verde-GO segundo resultados obtidos, 2012 (n=30)

\section{Discussão}

O leite materno é recomendado pelo Ministério da Saúde e Organização Mundial de Saúde como o melhor alimento para o lactente; contudo, a amamentação pode ser interrompida ou dificultada por questões fisiológicas, socioeconômicas, culturais ou emocionais. ${ }^{3,7,14}$

Tanto no início do aleitamento quanto em seu decorrer ou em caso de adoção, pode ser necessário utilizar estratégias para iniciar, retomar ou aumentar a produção de leite materno - nestes casos, o procedimento indicado pode ser a relactação. ${ }^{8-11}$

Em revisão de literatura sobre as taxas de sucesso da relactação no Brasil, Estados Unidos da América, Peru, Índia e Austrália, verificou-se que de 1.295 mulheres que fizeram a técnica de relactação, 1.136 obtiveram sucesso, representando $86,7 \%$ do total. ${ }^{12}$ 
O estudo de Alves, Figueira \& Nacul, ${ }^{15}$ realizado em ambiente hospitalar, obteve resultados semelhantes aos do presente trabalho, em que $27,6 \%$ de mães pernambucanas de um total de 163 obtiveram sucesso na relactação. Outro estudo, ${ }^{16}$ no entanto, apontou que $83 \%(n=116)$ das mães indianas de um total de 139 obtiveram sucesso com a técnica.

Características de contexto podem explicar, ao menos em parte, a diferença entre as taxas de sucesso encontradas nos diversos estudos. São exemplos disso as condições e ambientes onde as orientações são realizadas (se em ambiente hospitalar ou ambulatorial, onde a técnica possa ser demonstrada e realizada junto com as mães) e as características culturais e rede de apoio à amamentação em cada região de estudo. ${ }^{12,16}$ No presente estudo, a técnica era apenas explicada em um ambiente de sala administrativa, seja para mãe, pai ou outros, o que talvez possa explicar a menor taxa de resultados bem-sucedidos.

Um estudo em ambiente hospitalar com 200 mulheres no Egito realizou três abordagens diferentes para o aconselhamento da técnica e aferiu os resultados via acompanhamento telefônico. A taxa de sucesso da relactação variou de acordo com o tipo de abordagem utilizado. A estratégia educacional mais eficaz foi a resolução de problemas (45\%) e o treinamento (50\%). A menos eficaz foi a abordagem de advertência (5\%). Os determinantes da relactação identificados foram nível de escolaridade da mãe, utilização de copo para alimentação sem mamadeiras ou chupetas e o envolvimento do marido no processo. Apenas $10 \%$ conseguiram relactar, ${ }^{17}$ taxa inferior à do presente estudo, possivelmente pela baixa taxa de sucesso entre aquelas que receberam a abordagem de advertência, o que sugere o quanto uma abordagem acolhedora e com habilidades de comunicação pode ser importante para o sucesso da técnica. Cabe ressaltar que os familiares e entes próximos têm papel fundamental na prática da técnica de relactação, uma vez que motivam a mulher. ${ }^{12}$

No presente estudo, foi observada uma alegação comum, a de rejeição do peito pelo bebê. A dificuldade do bebê em pegar o peito consiste num grande desafio, por estar habituado a mamadeira. ${ }^{9}$ Além de a criança não saber sugá-lo, não o reconhece, rejeitando-o terminantemente. Ao tentar levá-lo ao peito, o bebê o empurra com as mãos, chorando e demonstrando que não quer.

O sucesso da relactação parece ser mais fácil quando o bebê tem menos de dois meses de vida, não está acostumado com bicos artificiais e apresenta menos tempo de interrupção da amamentação. ${ }^{9}$ Contudo, segundo a revisão realizada por Mariano, ${ }^{12}$ o intervalo de tempo sem amamentar ao seio tem mais influência que a idade do bebê no sucesso da amamentação. 
É necessário lembrar que os resultados deste relato de experiência são gerais e não consideram todas as características dos participantes, como idade do lactente, idade materna, participação e envolvimento familiar, suporte profissional, uso ou não de medicações ou outros dispositivos para facilitar a produção do leite, entre outros. De qualquer forma, os resultados aqui apresentados reforçam as evidências de que a relactação é uma possibilidade terapêutica para crianças com necessidades alimentares especiais.

Essa técnica é muito importante e deve continuar a ser incentivada em vários locais de atenção à saúde da criança. A promoção da amamentação resulta na boa saúde do bebê e previne diversas doenças, devido às inúmeras propriedades do leite materno, que além de ser nutritivo, anti-infeccioso e imunológico, requer baixo custo e promove a interação entre mãe e filho. ${ }^{13,18,19}$

Destaca-se ainda que, diante da complexidade da organização e da gestão de programas de dispensação de fórmulas nutricionais para lactentes no âmbito do Sistema Único de Saúde (SUS), é preciso que se tenha um olhar ampliado para além de suas portas. Identificar os possíveis pontos críticos na linha de cuidado à saúde da criança é primordial. Merece atenção a prática corriqueira nas maternidades públicas e privadas quanto a oferta de pré-lacteos, ${ }^{20}$ que acarretam aumento considerável do risco de desenvolver alergias alimentares, ${ }^{19,21}$ resultando na busca por programas de dispensação de fórmulas. É notório, também, o desconhecimento geral dos profissionais quanto ao diagnóstico das alergias e intolerâncias alimentares, ${ }^{21-23}$ e também sobre o manejo clínico da amamentação. ${ }^{24}$ Faz-se necessária, portanto, a atenção qualificada não apenas nas maternidades, mas durante o pré-natal nas unidades básicas de saúde, na rede de saúde suplementar ou privada.

É primordial a implantação de um programa de qualificação continuada e permanente dos profissionais para promoção e apoio ao aleitamento materno e alimentação complementar saudável em diversas esferas e pontos de atenção, bem como para qualificação profissional no manejo clínico das alergias e intolerâncias alimentares.

\section{Conclusão}

Os achados aqui apresentados contribuem para a discussão da problemática de prescrição e dispensação de fórmulas nutricionais no SUS, visto que as orientações de relactação podem, seguramente, fazer parte das escolhas terapêuticas de médicos pediatras, enfermeiros, nutricionistas, fonoaudiólogos e outros profissionais da saúde que lidam com a problemática do desmame precoce. 
A amamentação traz inúmeros benefícios à saúde do bebê, além de reduzir gastos diretos e indiretos com saúde. Portanto, os órgãos executivos municipais devem reconhecer que é muito mais vantajoso oferecer assistência adequada para incentivo à amamentação às nutrizes e sua rede de apoio, do que fornecer fórmulas infantis.

Na experiência relatada, uma parcela expressiva das mães obteve sucesso com a relactação. Contudo, percebe-se que, apesar da orientação dessa técnica, as mães encontraram dificuldades que interferiram no resultado final, impedindo a produção de leite. O sucesso talvez possa ser ampliado com uma rede de apoio e incentivo à amamentação, o que possibilitaria o aumento de práticas bem-sucedidas e de baixo custo para a saúde pública como esta.

A continuidade das ações constitui um dos maiores desafios para o SUS. A alta rotatividade dos profissionais da gestão e os vínculos empregatícios instáveis tornam-se dificultadores para a manutenção das ações e exigem qualificação permanente para que se tenha uma equipe qualificada, sensibilizada e motivada.

O PAN tem tido uma atuação positiva no enfrentamento das prescrições abusivas, e por vezes desnecessárias, de fórmulas infantis e do desestímulo do aleitamento materno e tenta reverter essa situação mediante a qualificação profissional para motivar o restabelecimento do aleitamento materno nas famílias, por meio da técnica de relactação. Novos investimentos são necessários para a qualificação profissional permanente, com utilização de recursos financeiros para isso, dentre eles os provenientes do Financiamento em Alimentação e Nutrição (FAN/MS).

\section{Agradecimentos}

À colega de trabalho Waleska Fonseca Campos Oliveira por sempre ter sido apoiadora dessa e de outras estratégias, aos estagiários na época de realização da pesquisa Márcio Borges Dias e Debora Fernanda Sartori. À Coordenação de Saúde da Criança e a Coordenação de Vigilância Nutricional do estado de Goiás pelos cursos de formação primordiais. E às mães, pais e apoiadores por terem contribuído para o registro dessa atividade. 


\section{Referências}

1. Instituto Brasileiro de Geografia e Estatística. Censo demográfico 2010: resultados divulgados no Diário Oficial da União em 04.11.2010 [Internet]. Disponível em: http://www.ibge.gov.br/home/ estatistica/populacao/censo2010/resultados_dou/default_resultados_dou.shtm

2. Carvalho MR. Planejando cidades amigas da amamentação. Rev. IBAM 1997; 44(220):50-62.

3. Carvalho MR. Manejo ampliado da amamentação: o aleitamento materno sob a ótica da saúde coletiva. In: Carvalho MR, Tavares LAM. Amamentação: bases científicas. 3 ed. Rio de Janeiro: Guanabara Koogan; 2010. p. 328-345.

4. World Health Organization. Infant and young child feeding: model chapter for textbooks for medical students and allied health professionals. Geneva: WHO; 2009. 99 p.

5. Brasil. Ministério da Saúde. Rede amamenta Brasil: o caderno do tutor. Brasília: Ministério da Saúde; 2009. 118 p.

6. Brasil. Ministério da Saúde. ENPACS: Estratégia nacional para alimentação complementar saudável: caderno do tutor. Brasília: Ministério da Saúde; 2010. 108 p.

7. Brasil. Ministério da Saúde. Dez passos para uma alimentação saudável: guia alimentar para crianças menores de dois anos: um guia para o profissional da saúde na Atenção Básica. 2. ed. Brasília: Ministério da Saúde; 2010. 72 p.

8. World Health Organization. Relactation: review of experience and recommendations for practice. Geneva: WHO; 1998. 42 p.

9. Melo SL. Amamentação: contínuo aprendizado. 2 ed. São Paulo: All Print; 2010. 258 p.

10. Sanches MTC. Enfoque fonoaudiológico. In: Carvalho MR, Tavares LAM. Amamentação: bases científicas. 3 ed. Rio de Janeiro: Guanabara Koogan; 2010. p.101-122.

11. Tamez RN. Atuação de enfermagem. In: Carvalho MR, Tavares LAM. Amamentação: bases científicas. 3 ed. Rio de Janeiro: Guanabara Koogan; 2010. p. 123-136.

12. Mariano GJS. Relactação: identificação de práticas bem sucedidas. Revista de Enfermagem 2011; 3(3):163-170.

13. Bordalo JD. Aleitamento materno: relactação e lactação induzida [dissertação]. Covilhã: Universidade da Beira Interior; 2008.

14. Butte NF, Lopez-Alarcon MG, GARZA C. Nutrient adequacy of exclusive breastfeeding for the term infant during the first six months of life. Geneva: WHO; 2002. 47 p.

15. Alves JG, Figueira F, Nacul LC. Relactation improves nutritional status in hospitalized infants. Journal of Tropical Pediatrics 1999; 45(2):120-121.

16. De NC, Pandit B, Mishra SK, Pappu K, Chaudhuri SN. Initiating the process of relactation: an institute based study. Indian Pediatrics 2002; 39(2):173-178.

17. Abul-Fadl AMA, Kharboush I, Fikry M, Adel M. Testing communication models for relactation in an egyptian setting. Breastfeeding Medicine 2012; 7(4):248-254. 
18. Martins Filho J, Sanged CAA, Brenelli MA, Rodrigues MTM, Ferreira MRGB, Mathiazzi TAF, et al. Relactação - I. Proposta de uma técnica para facilitar a estimulação da lactação. Pediatria 1981; 3(1):319-329.

19. Brasil. Ministério da Saúde. Saúde da criança: nutrição infantil: aleitamento materno e alimentação complementar. Brasília: Ministério da Saúde; 2009. 112 p.

20. Segall-Corrêa AM, Marín-León L, Panigassi G, Rea MF, Pérez-Escamilla R. Amamentação e alimentação infantil. In: Brasil. Ministério da Saúde. Centro Brasileiro de Análise e Planejamento. Pesquisa nacional de demografia e saúde da mulher e da criança - PNDS 2006: dimensões do processo reprodutivo e da saúde da criança. Brasília: Ministério da Saúde; 2009. p. 195-212.

21. Ferreira CT, Seidman E. Alergia alimentar: atualização prática do ponto de vista gastroenterológico. J. Pediatr. 2007; 83(1):7-20.

22. Dirceu S, Jacb CM, Pastorino AC, Porto Neto A, Burns DA, Sarinho ESC, et al . O conhecimento de pediatras sobre alergia alimentar: estudo piloto. Rev. Paul. Pediatr. 2007; 25(4):311-316.

23. Cortez APB, Medeiros LCS, Speridião PGL, Mattar RHGM, Fagundes Neto U, Morais MB. Conhecimento de pediatras e nutricionistas sobre o tratamento da alergia ao leite de vaca no lactente. Rev. Paul. Pediatr. 2007; 25(2):106-113.

24. Caldeira AP, Aguiar GN, Magalhães WAC, Fagundes GC . Conhecimentos e práticas de promoção do aleitamento materno em Equipes de Saúde da Família em Montes Claros, Minas Gerais, Brasil. Cadernos de Saúde Pública 2007; 23(8):1965-1970.

Recebido: 15/4/2014

Revisado: 02/6/2014

Aprovado: 07/7/2014 
